\title{
Highly efficient, all-organic bioluminescence-photosensitizer conjugate eradicates early-stage tumors and prevents metastasis in mice
}

\author{
Hao Yan', Sarah Forwad', Kwon-Hyeon Kim', Yue Wu', Jie Hui', Anokhi Kashiparekh', \\ Seok-Hyun Yun ${ }^{1 *}$
}

\author{
${ }^{1}$ Harvard Medical School and Wellman Center for Photomedicine, Massachusetts General Hospital, \\ 65 Landsdowne St., UP-5, Cambridge, MA 02139, USA \\ *e-mail: syun@hms.harvard.edu
}

Photodynamic therapy (PDT) is an established treatment modality using light-activatable drugs. Despite its unique cytotoxic mechanism, the shallow penetration of light has been a serious drawback limiting the applications of PDT. Here, we report bioluminescence-activated PDT (BL-PDT) using efficient bioluminescence resonance energy transfer (BRET) conjugates of clinically approved photosensitizers, Ce6, and luciferase proteins. A high photon-to-Ce6 conversion efficiency (80\%), along with intracellular delivery by membrane-fusion liposomes, enabled effective cancer cell killing in vitro. In a syngeneic mouse model of aggressive 4T1 triplenegative breast cancer, as well as a xenograft model of MDA-MB-231 tumors, BL-PDT resulted in complete tumor remission and prevention of metastasis, as well as neo-adjuvant effects. Our result shows the promise of molecularly activable, clinically viable, depth-unlimited phototherapy.

\section{Introduction}

Photodynamic therapy (PDT) is a clinically approved treatment modality for several diseases including cancers $^{1-2}$. PDT uses light and photosensitizers that, upon absorbing photon energy, can generate reactive oxygen species (ROS) such as single oxygen. The ROS leads to various therapeutic effects, such as the killing of malignant cells, destruction of angiogenic blood vessels, and activation of antitumor immune responses. Effective PDT critically relies on sufficient light dose at target tissues, typically requiring an optical fluence of $\sim 50 \mathrm{~J} / \mathrm{cm}^{2}$ at the surface of tissue. This amount of dose can produce PDT effects at tissue depths up to a few millimeters below the surface ${ }^{3-}$ 7. This shallow depth has been an important limitation of PDT, constraining their clinical applications.

To overcome the limited therapeutic depths, a technique to generate light within tissue by Cherenkov radiation has been proposed ${ }^{8}$. However, its use of high-energy radiation and inorganic $\mathrm{TiO}_{2}$ nanoparticles raise safety concerns for clinical translation. Another strategy replacing the external light illumination of conventional PDT is using chemiluminescence or bioluminescence $(B L)$ as internal light sources ${ }^{9-11}$. Although the molecular 
luminescence has much weaker intensity than what external irradiation can provide, efficient Förster resonance energy transfer from the luminescent group to photosensitizers can, in principle, compensate for the intensity difference and produce substantial PDT effects. Recently, a combination of a chemiluminescent donor, luminol, and a photosensitizer as the acceptor was shown to produce efficient ROS-induced cell death in vitro and tumor growth inhibition in vivo ${ }^{9-10}$. However, luminol requires endogenous $\mathrm{H}_{2} \mathrm{O}_{2}$ at a high concentration in the target tissue. This, along with its high affinity to serum albumin and DNA, raises biosafety concerns ${ }^{11-12}$. Compared to chemiluminescence with low quantum yields $(<0.1)$, bioluminescence using luciferin / luciferase as the donor offers higher quantum yields of $>0.4 .{ }^{13-14}$ Bioluminescence resonance energy transfer (BRET) to Rose Bengal ${ }^{15}$ and photosensitive fusion proteins has been explored ${ }^{16}$, but their low activation efficiencies limit cell-killing effects. BRET using inorganic quantum dots can be more efficient but is not suitable for clinical translation due to the toxic quantum dot materials.

Here we report highly efficient BRET-induced PDT enabled by a novel photosensitizer agent consisting of clinically used photosensitizers Chlorin e6 (Ce6) and Renilla reniformis Luciferase 8 (RLuc8) proteins. An optimized RLuc8 to Ce6 ratio (1:25) and excellent spectral matching between BL emission and Ce6 absorption ${ }^{9-10}$ allowed us to achieve a high activation efficiency — probability of producing an activated Ce6 per BL photon - of $80 \%$. We show that fusogenic nano-liposomes are a highly effective vehicle to deliver the conjugates into cancer cells. We compare the therapeutic effects of our BL-PDT technique with conventional PDT for 4T1 murine and MDA-MB231 human triple-negative breast cancer (TNBC) cells in vitro and then in vivo using mouse models. With one-time injection of Luc-Ce6, we demonstrate complete remission of the tumor and prevention of metastasis in lymph nodes and lungs. We also evaluate the neo-adjuvant effect of intratumoral BL-PDT for advanced tumors.

\section{Results}

Synthesis and BRET efficiency of Luc-Ce6 conjugate

Figure 1a illustrates the working principle of the Luc-Ce6 conjugate. Briefly, we prepared a Ce6 derivative with 6amino hexanoic acid amide, termed Ce6-HA (710 Da). Ce6-HA molecules are conjugated to RLuc8 proteins ( 37 $\mathrm{kDa}$ ) using EDC/Sulfo-NHS chemistry (Fig. S1). One RLuc8 protein has a total of 311 amino acid residues ${ }^{17-18}$. The conjugation is formed between the active amino residues and the acid amide of the Ce6-HA, resulting in Ce6 molecules conjugated on the surface of RLuc8. When a substrate Me-eCTZ (briefly CTZ hereinafter) binds with the RLuc8 (briefly Luc8), the BL reaction can occur, producing optical energy, which would otherwise radiate as a photon but in this case, is desired to be transferred to one of the Ce6 molecules via BRET. The excited Ce6 then may react with an oxygen molecule nearby and convert it to singlet oxygen $\left({ }^{1} \mathrm{O}_{2}\right)$. This probability of ROS generation from activated Ce6 is $65-70 \%$ in typical in vitro and in vivo conditions ${ }^{19-20}$.

A mixture of Luc8 and CTZ in solution emits BL photons with a center at $415 \mathrm{~nm}$, which overlaps well with the absorption peak of Ce6 at $406 \mathrm{~nm}$ (Fig. 1b). When Luc8 (0.3 $\mu \mathrm{M})$ andCe6-HA (7.5 $\mu \mathrm{M})$ were simply mixed in solution 
with CTZ, fluorescence from Ce6 at $660 \mathrm{~nm}$ appeared (Fig. 1b), due to the absorption of BL photons by Ce6 and BRET from Luc8/CTZ to Ce6 molecules in proximity $(<5 \mathrm{~nm})$ to each other. We fabricated Luc-Ce6 conjugates with different ratios of Ce6 to Luc8. As the ratio increased from 1:1 to 15:1, the BL intensity at $440 \mathrm{~nm}$ decreased, and the fluorescence intensity at $660 \mathrm{~nm}$ increased. And it had a plateau at ratios between 15 and 28 (Fig. 1c). We determined the optimal conjugation ratio to be $25: 1$ and used this value in subsequent experiments. A simple kinetic model considering a BRET efficiency and hindrance of Ce6 to the interaction of Luc explained the experimental result reasonably well (dashed lines in Fig. 1d).

Figure 1e shows the representative emission spectrum of Luc-Ce6 conjugates ( $0.3 \mu \mathrm{M})$, integrated over $1 \mathrm{~min}$ after injecting a non-saturating small amount of CTZ $(\sim 1 \mu \mathrm{M})$. The BL spectra from RLuc8 and a simple mixture of Ce6 and RLu8 with the ratio of 25 are also shown. The BRET ratio, defined by the ratio of total acceptor emission $(B, 600-800 \mathrm{~nm})$ to the total donor emission $(A, 400-600 \mathrm{~nm})$, was 0.71. Quantitative absorption (Fig. S2b) and fluorescence (Fig. S3b) measurements confirmed the average number of Ce6 in the Luc-Ce6 conjugate to be about 25 as intended. The probability of BL energy ending up exciting a Ce6 molecule can be calculated by using a typical Ce6 fluorescence quantum yield of $15 \%{ }^{21}$. The ratio of the number of activated Ce6 molecules to the number of BL photons that are generated without Ce6 conjugation was determined to be $80 \%$ (Table S1). This is a remarkably high efficiency of excitation. For far-field illumination, the absorption cross-section of Ce6 is $\sim 1 \times$ $10^{-15} \mathrm{~cm}^{2}$ at $405 \mathrm{~nm}$. This means $10^{15}$ photons $/ \mathrm{cm}^{2}$ are needed to excite a single Ce6 molecule with a probability of $37 \%$ (1/e). In fact, the great majority of photons supplied by far-field illumination in conventional PDT end up not being absorbed by photosensitizers. By contrast, $80 \%$ of BL photon is used to excite Ce6.

We used a singlet oxygen sensor green (SOSG) dye ${ }^{22}$ to detect ${ }^{1} \mathrm{O}_{2}$ generated by Luc-Ce6 and CTZ in solution. The SOSG fluorescence intensity increased with an increasing amount of CTZ (square, Fig. 1f). In a separate experiment, we measured BL intensity to reach a plateau when the ratio of CTZ to Luc8 exceeded 100 (diamond, Fig. 1f). The result was compared with the SOSG fluorescence after conventional laser-PDT using Ce6-HA as the photosensitizer and $405 \mathrm{~nm}$ laser irradiation with an intensity of $125 \mathrm{~mW} / \mathrm{cm}^{2}$ for $8 \mathrm{~s}$ (Fig. $1 \mathrm{~g}$ and Fig. S5). In terms of singlet oxygen generation, $280 \mu \mathrm{M} \mathrm{CTZ}$ on $2.8 \mu \mathrm{M}$ Luc-Ce6 is equivalent to laser fluence of $1 \mathrm{~J} / \mathrm{cm}^{2}$ on $10 \mu \mathrm{M}$ Ce6.

Intracellular delivery of Luc-Ce6 conjugates

Intracellular delivery of protein constructs has been challenging ${ }^{23-25}$. We found fusogenic nano-liposomes ${ }^{24}$ (Fusolip) to be more effective than conventional liposomal delivery (Fig. S6 and Fig. S7). Upon fusing into the cellular membrane, the liposome can directly inject its cargo into the cytoplasm (Fig. 2a). This direct delivery escapes from the typical endocytosis process in which the protein cargo is confined and rapidly degraded in lysosomes ${ }^{26-27}$. We loaded the Luc-Ce6 conjugate in Fuso-lip using charge differential drive followed by membrane extrusion through $100 \mathrm{~nm}$ pores. Using dynamic light scattering, we measured the hydrodynamic size of Luc-Ce6 to be 
$17 \mathrm{~nm}$, Fuso-lip to be $70 \mathrm{~nm}$, and Luc-Ce6/Fuso-lip complex to be $\sim 100 \mathrm{~nm}$ (Fig. 2b). The final Luc-Ce6/Fuso-lip complex had a zeta potential of $-17.8 \mathrm{mV}$ (Fig. S8).

We tested intracellular delivery with $4 \mathrm{~T} 1$ murine breast cancer cells. Figure 2c shows fluorescence confocal images of 4T1 cells after 30 min incubation with Luc-Ce6/Fuso-lip (Fig. S9). Fuso-lip fuse immediately with the cell membrane. Luc-Ce6 conjugates are distributed in the cytosol but not in the nucleus. The cells incubated with $5 \mu \mathrm{M}$ Luc-Ce6 (containing $75 \mu \mathrm{M}$ Ce6) in Fuso-lip exhibited stronger Ce6 fluorescence than cells incubated with $25 \mu \mathrm{M}$ Ce6-HA for 120 min incubation time. Flow cytometry analysis supported the effectiveness of Luc-Ce6 delivery by Fuso-lip, nearly comparable to the intracellular uptake of small Ce6-HA molecules (Fig. 2d). The intracellular distribution of Luc-Ce6 was not correlated with the locations of lysosomes (Figs. 2e, S10, and S11). This supports that the uptake mechanism of Luc-Ce6/Fuso-lip escapes from typical endocytosis and does not involve the endosomal-lysosomal system ${ }^{28-29}$. Ce6 molecules are known to accumulate in the mitochondria membrane after entering cells $s^{30-31}$ partly because of their hydrophobic aromatic rings. This association with mitochondria is thought to be important for high cytotoxicity of $\mathrm{PDT}^{32}$. We observed an overlap of mitochondria with Ce6 and Luc-Ce6 (Figs. 2f, S12, and S13), which also coincide with the fluorescence signal from DCFDA, an intracellular ROS sensor, in both laser-PDT (Fig. S14) and BL-PDT (Fig. S15). Given the similar intracellular distributions, we expect similar cytotoxic mechanisms between BL-PDT and laser-PDT using Ce6.

In vitro BL-PDT on 4T1 and MDA-MB-231 cells

We evaluated the intrinsic cytotoxicity of CTZ, Fuso-lip, and Luc-Ce6/Fuso-lip reagents on 4T1 (murine) and MDAMB-231 (human) TNBC cell lines and, also, L929 (mouse fibroblast) cells in culture using a Cell Counting Kit-8 (CCK8) assay (Fig. S16). No significant cytotoxicity was observed from all three cell types for $48 \mathrm{~h}$ even with high concentrations of Luc-Ce6 (10 $\mu \mathrm{M})$ and CTZ (100 $\mu \mathrm{M})$.

Using the DCFDA intracellular assay, we detected considerable ROS generation in 4T1 cells after BL-PDT (10

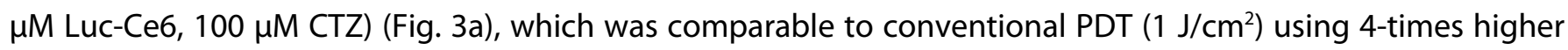
Ce6 amount (Fig. 3a). The DCFDA signal increased with increasing amount of Luc-Ce6 for a fixed amount of CTZ (Figs. 3b, S17, and S18). BL-PDT resulted in near complete cell killing confirmed by live/dead fluorescence dyes and morphological changes (Figs. 3c and S19-21). Cell killing by laser-PDT occurs only in regions illuminated with sufficient laser energy (Figs. 3c and S21). When we placed layers of chicken breast tissues on top of cell samples in the optical path, simulating deep tissue therapy, no phototoxicity was observed even with a modest tissue thickness of $300 \mu \mathrm{m}$ (Fig. S22). Using the CCK-8 assay, we compared the dose dependent cytotoxicity (Figs. 3d and 3e). The half-maximal inhibitory concentration $\left(\mathrm{IC}_{50}\right)$ values of Luc-Ce6 in BL-PDT were measured to be 5.6 $\mu \mathrm{M}$ for $4 \mathrm{~T} 1$ cells and $1.5 \mu \mathrm{M}$ for MDA-MB-231 cells. By comparison, IC 50 values of Ce6 in laser-PDT were $21 \mu \mathrm{M}$ for 4T1 and 5.4 $\mu \mathrm{M}$ for MDA-MB-231 cells, respectively. Moreover, we used an Annexin V-FITC/PI apoptosis detection kit and flow cytometry to confirm apoptotic death of 4T1 cells 24 hours after BL-PDT and laser-PDT (Fig. S24). 


\section{Tumor cell killing by intratumoral BL-PDT in vivo}

For preclinical testing, we first established a metastasis TNBC orthotopic model, using an injection of $\sim 10^{6} 4$ T1 cells into the mammary fat pad of a BALB/c mouse. The 4T1 mammary carcinoma is highly tumorigenic and invasive and, unlike most tumor models, can spontaneously metastasize from the primary tumor to multiple distant sites. The implanted tumor grew rapidly, reaching 4-5 mm in size in 1 week and 9-12 mm in 3 weeks (Fig. S25). Tumor invasion into surrounding tissues was observed at Day 14 (Fig. S25C). Consistently, no apparent metastasis was found in the sentinel lymph node (SLN) and lung at Day 7. On Day 14, small metastases were detected in the SLN and lung (Fig. S26 and Fig. S27). On Day 21, multiple metastatic foci were evident throughout the lung. From this result, we determined that early metastasis occur between 1 and 2 weeks.

We assessed the effects of BL-PDT using a protocol depicted in Fig. 4a. On day 14 after tumor inoculation, LucCe6/Fuso-lip ( $2.5 \mathrm{mg} / \mathrm{kg}$ ) was intratumorally injected into the tumor at several sites using a fine needle. To confirm intratumoral distribution, we harvested tumors $6 \mathrm{~h}$ after an intratumoral injection of Luc-Ce6/Fuso-lip without CTZ injection and found the reagent distributed extensively in the tumor (Figs. 4b, S28, and S29). For mice in the treatment group, following a Luc-Ce6/Fuso-lip injection we waited for $6 \mathrm{~h}$ for the Luc-Ce6 to be delivered to the tumor cells and then intravenously injected the first dose of CTZ $(100 \mu \mathrm{M}, 50 \mu \mathrm{l})$. Then, 5 additional injections of CTZ with the same dose were given in subsequent $24 \mathrm{~h}$ (Fig. 4a). The tumors were harvested at 3 days after the treatment was completed. Their frozen tissue sections were examined using the terminal deoxynucleotidyl transferase dUTP nick-end labeling (TUNEL) assay to detect cell apoptosis. The BL-PDT treated groups showed strong TUNEL signals over larger areas (Fig. 4c). PBS-treated and Luc-Ce6-sham-treated tumors had no obvious TUNEL signals (Figs. 4d and S30). Laser-PDT treated groups, which had received the same intratumoral injection of Luc-Ce6/Fuso-lip and treated with 405-nm laser $\left(90 \mathrm{~J} / \mathrm{cm}^{2}\right)$ instead of CTZ, showed TUNEL signals only in superficial regions along the tumor boundary within $<1 \mathrm{~mm}$ depth from the surface (Fig. 4e). This result showed the advantage of BL-PDT over laser-PDT in terms of therapeutic depth.

\section{In vivo antitumor treatment by BL-PDT}

Having confirmed the cell killing effect in vivo, we tested BL-PDT on tumors in their early stage of development. Figure 5a depicts the treatment schedule. BL-PDT was applied on tumors 7 days after 4T1 cell inoculation, a time point when no metastasis is expected to have occurred. For BL-PDT treated mice $(n=5)$, we measured an immediate shrinkage of the tumor volume within 2 days after treatment. A complete tumor regression was achieved by Day 18, without relapse (Fig. 5c). The body weights of the treated mice were normal (Fig. 5 d). In stark contrast, laser-PDT treated mice showed nearly unaffected, exponential growth curves (Fig. 5b and 5c). Various organs were harvested at Day 25. Histology of tissue sections from the heart, liver, kidney, and spleen showed no sign of tissue damage (Fig. S31), nor metastasis (Figs. 5f-g and S32). At Day 25, all the control, sham, and laser- 
PDT treated mice showed substantial metastasis to the SLN and lung (Figs. 5e-g). Our result showed that BL-PDT can eradicate early-stage tumors and prevent metastasis in mice.

\section{Neoadjuvant BL-PDT}

Surgery is the gold standard treatment for removing primary and large tumors. Neoadjuvant chemotherapy and radiation therapy are often performed before surgery to downstage the tumors and reduce the extensiveness of surgery and postoperative complications ${ }^{33-35}$. We tested the neoadjuvant potential ${ }^{36-38}$ of BL-PDT (Fig. 6a). We injected Luc-Ce6/Fuso-lip at the boundary of primary tumor on Day 14, a time point when 4T1 cells typically have started invasion (Fig. 6b). Immediately after the injection, Luc-Ce6 and Fuso-lip are mainly localized in the tumor boundary (Fig. S33). 5 days after the completion of BL-PDT, the tumors were collected for histological analysis. Most tumor cells in the border appeared to be round, atrophic, and apoptotic (Figs. $6 \mathrm{c}$ and S34). This morphological change visualized the margin more vividly than tumors in control animals (Fig. 6d) in which the tumor invasion continued to have progressed. Tissues in the laser-PDT treated group also showed blurred tumor boundaries (Fig. S35). Our result shows that adjuvant BL-PDT can shrink the tumor size and delineate the tumor margin.

\section{Discussion}

The optimized luciferase-photosensitizer BRET conjugates, along with membrane-fusion liposomal delivery, enabled us to demonstrate highly effective BL-PDT for early-stage treatments of TNBC. The molecularly activatable Luc-Ce6 conjugate enables depth-independent PDT, a major advantage over conventional lightactivatable photosensitizers. The toxicity of the all-organic reagent is minimal to none until CTZ is administered. This triggerable action allows BL-PDT to be performed at an optimal time when the agents have been delivered to target regions for the best therapeutic outcome. It could also help achieving low background cytotoxicity, an advantage over non-activable drugs such as conventional chemotherapy agents.

The ROS-mediated mechanism makes PDT a unique modality different from chemotherapies, radiation therapies, and immunotherapies ${ }^{39-41}$. Our results demonstrated the potential of BL-PDT as a standalone, complementary, or synergistic treatment option for cancer treatment. In this study, we have investigated intratumoral delivery by local injection of Luc-Ce6/Fuso-lip. Although this method could be viable for treating small tumors or neo-adjuvant treatment of larger tumors, as demonstrated, it may be a worthwhile to develop a systemic delivery method of BRET conjugates for broader applications such as treating metastases ${ }^{42-45}$. It will also be interesting to develop other combinations of luciferase, luciferin, and photosensitizer than Luc-Ce6-CTZ, with possible differences in target tissues and organelles. BL-PDT can be applicable to treating non-cancers, such as deep skin lesions. Finally, the BRET strategy may be extended to other phototherapies, such as antibacterial blue 
light therapies ${ }^{46-47}$, that use endogenous photosensitizers. Our promising results encourages further developments toward clinical BRET-based phototherapies.

\section{Materials and Methods}

Synthesis of Luc-Ce6 conjugate. The Luc-Ce6 conjugate was synthesized by directly conjugating Renilla luciferase 8 (Rluc8, or simply Luc, G-Biosciences) with chlorin e6 mono 6-amino hexanoic acid amide (Ce6-HA) molecules (Santa Cruz Biotechnology) using the standard N-(3-dimethyl aminopropyl)-N-ethyl-carbodiimide hydrochloride (EDC)/N-hydroxysulfosuccinimide (Sulfo-NHS)-activated condensation reaction. Briefly, 1 mg Ce6$\mathrm{HA}\left(1.4 \times 10^{-6} \mathrm{~mol}\right)$ powder was thoroughly dispersed in $100 \mu \mathrm{l}$ dimethyl sulfoxide (DMSO) and then mixed with $400 \mu \mathrm{l}$ DPBS buffer to make a $0.5 \mathrm{ml}, 2 \mathrm{mg} / \mathrm{ml}$ solution. Then, $100 \mu \mathrm{IEDC}\left(2.6 \times 10^{-5} \mathrm{~mol}\right)$ and $50 \mu \mathrm{l}$ Sulfo-NHS (4.6 $\times 10^{-6} \mathrm{~mol}$ ) were added into a Ce6-HA solution and reacted for $20 \mathrm{~min}$ at room temperature. After this crosslinking reaction, $447 \mu \mathrm{l}$ 2-mercaptoethanol ( $55 \mathrm{mM}$ ) was added to the solution to quench the excess EDC, and the sample was incubated for $10 \mathrm{~min}$ at room temperature. For conjugation, a new modified Ce6-HA solution was added into a Rluc8-DPBS solution, gently pipetted several times, and then reacted for $3 \mathrm{~h}$ in an Eppendorf ThermoMixer-C instrument (300 rpm) at room temperature. The ratio of Ce6-HA and Rluc8 was varied to test different mole conjugation ratios. After conjugation, the mixture solution was filtered using Zeba spin desalting columns (7K MWCO) to eliminate unreacted small molecules and obtain the final Luc-Ce6 DPBS solution.

Characterization of Luc-Ce6 conjugate. An electron-multiplication charge-coupled device (EMCCD)-equipped spectrometer (Newton, Andor) was used to measure bioluminescence (BL) spectra from various solutions. To measure BL spectra from RLuc8 and methoxy e-coelenterazine (Me-eCTZ, or simply CTZ), $1 \mathrm{ml}, 0.3 \mu \mathrm{M}$ CTZ was added to a $1 \mathrm{ml}, 0.3 \mu \mathrm{M}$ Luc-Ce6 DPBS solution in a polystyrene cuvette. The BL emission was collected with $0.1 \mathrm{~s}$ exposure and 300 frame averaging. The fluorescence spectra of Ce6-HA solutions (from 1.7 to $24.9 \mu \mathrm{M}$ ) and LucCe6 solution $(0.38 \mu \mathrm{M})$ were measured by using the EMCCD-coupled spectrometer and a xenon lamp filtered by a monochromator. A microplate reader (Epoch 2, Biotek Instruments) in the spectrophotometer mode was used to measure the absorption spectra of Ce6-HA at different concentrations (from 8.3 to $166 \mu \mathrm{M}$ ) and of Luc-Ce6 conjugates (1.39 and $2.78 \mu \mathrm{M}$ ). A dynamic light scattering instrument (Malvern Zetasizer Nano-ZS, $633 \mathrm{~nm}$ ) was used to measure the hydrodynamic size and Zeta potential.

Detection of singlet oxygen in solution. A singlet oxygen sensor green dye (SOSG, Thermo Fisher Scientific), which is highly selective for ${ }^{1} \mathrm{O}_{2}$, was used to measure the amount of singlet oxygen in solution. A mixture solution of Luc-Ce6 or Ce6-HA with SOSG (dissolved in methanol) was prepared (10 $\mu \mathrm{M}$ SOSG in the mixture), and CTZ with different molar ratios (20 to 150) was added to BL-PDT sample solutions. The laser-PDT sample solutions were illuminated with $405 \mathrm{~nm}$ laser beam at $1 \mathrm{~J} / \mathrm{cm}^{2}$. The fluorescence spectra of the samples were measured 30 min after treatments.

Fusogenic nano-liposomes (Fuso-lip) and Luc-Ce6 loading. The two main components of liposomes, 1,2dioleoyl-3-trimethylammonium-propane (DOTAP) and 1,2-dioleoyl-sn-glycerol-3-phosphoethanolamine (DOPE), were purchased from Avanti Polar Lipids (stored at $-80^{\circ} \mathrm{C}$ ). A $1 \mathrm{mg} / \mathrm{ml}$ stock solution of DOPE/DOTAP with a weight ratio of 1:1 was prepared. After evaporating the chloroform solvent, DOPE and DOTAP were resuspended in DPBS buffer at $1 \mathrm{mg} / \mathrm{ml}$ concentration. For Luc-Ce6 loading, a $500 \mu \mathrm{l}, 27.8 \mu \mathrm{M}$ fresh solution of Luc-Ce6 was added into an $800 \mu \mathrm{l}$ DOPE/DOTAP solution, stirred vigorously for $10 \mathrm{~min}$ (300 rpm), and incubated in an 
ultrasonic bath (Elmasonic, $37 \mathrm{kHz}, 30 \%$ power) for $5 \mathrm{~min}$ at room temperature. To reduce the size of conjugateloaded liposomes, a mini-extruder instrument (Avanti Polar Lipids) was used to gently extrude the Luc-Ce6/Fusolip solution several times through membrane filters with $100 \mathrm{~nm}$ pore sizes for in vitro experiments or $50 \mathrm{~nm}$ pore sizes for in vivo experiments. After extrusion, 3,3'-dihexadecyloxacarbo-cyanine perchlorate (DiO) or 1,1'dioctadecyl-3,3,3',3'-tetramethyl-lindotricarbocyanine iodide (DiR) was added into the liposome solution with a ratio of $1 / 15(\mathrm{w} / \mathrm{w})$ for fluorescent staining of the liposomes. The sample was diluted with DPBS buffer to a desired working concentration.

Cell culture and in vitro cytotoxicity evaluation. Murine fibroblast L929 cell line cells, murine TNBC 4T1 cells, and human TNBC MDA-MB-231 cells were purchased from ATCC (American Type Culture Collection). Cells were cultured in recommended standard conditions. DMEM basic cell media was supplemented with fetal bovine serum $(10 \%, v / v)$ and penicillin/ streptomycin (1\%, v/v), known as "complete media". Cells were incubated at 37 ${ }^{\circ} \mathrm{C}$ in a humidified atmosphere of $5 \% \mathrm{CO}_{2}$ and $95 \%$ air. For the cytotoxicity studies, cells were seeded on a 96-well plate $(\sim 5,000$ cells per well) with $100 \mu$ complete media and cultured for $24 \mathrm{~h}$ prior to adding different test reagents: CTZ (25, 50, and $100 \mu \mathrm{M})$, Fuso-lip (50, 75, 100, and $150 \mu \mathrm{g} / \mathrm{ml})$, or Luc-Ce6/Fuso-lip (0.75, 1.25, 1.7, 2.5, 3.3, 5.0, 6.7, and $10 \mu \mathrm{M}$ ). After incubation for $4 \mathrm{~h}$ for CTZ and $30 \mathrm{~min}$ for Fuso-lip and Luc-Ce6/Fuso-lip, the cells were washed two times by DPBS buffer and cultured in fresh cell media for 24 or $48 \mathrm{~h}$. The cell viability was measured by using a cell counting kit-8 (CCK-8, ApexBio) assay. The absorbance was measured by using a spectrophotometer (Epoch 2, Biotek Instruments).

Intracellular delivery of Luc-Ce6 conjugate. Cells were plated in glass-bottomed well plates with the same density. After culturing for $24 \mathrm{~h}$, the cell media was removed, a Luc-Ce6/Fuso-lip DPBS solution was added, and the cells were incubated for $30 \mathrm{~min}$. After removing the Luc-Ce6/Fuso-lip solution, the cells were washed three times and cultured in fresh cell media for further experiments or fixed by $4 \%$ paraformaldehyde (PFA) for imaging. As a control group, Ce6-HA solution was added to the culture plate and incubated for $2 \mathrm{~h}$ before fixing with $4 \%$ PFA. The cell nuclei were stained with 4',6-diamidino-2-phenylindole (DAPI). A lyso-tracker probe (Invitrogen, Red DND-99) was used to stain intracellular lysosomes. $1 \mathrm{mM}$ probe stock solution was diluted to $1 \mu \mathrm{M}$ in DPBS buffer and added to the cells in glass-bottom plates to reach $50 \mathrm{nM}$ in the media. After $1 \mathrm{~h}$ incubation, the cells were washed 3 times with DPBS buffer, fixed with 4\% PFA, and examined with fluorescence confocal microscopy. A mitochondria tracker Red CMXRos dye (Invitrogen) and 2',7'-dichlorodihydrofluorescein diacetate (DCFDA, Cell Biolabs) were used for mitochondrial and intracellular ROS staining, respectively. The Mito-Tracker probe was dissolved in DPBS buffer to prepare $50 \mathrm{nM}$ solution. DCFDA was dissolved in DMSO to form a stock solution (500 $\mu \mathrm{M})$ and diluted into the Mito-Tracker DPBS buffer to obtain a $5 \mu \mathrm{M}$ working solution. After incubation with LucCe6/Fuso-lip, 4T1 cells in a glass-bottom plate were washed twice with DPBS and added with the Mito-Tracker /DCFDA-DPBS mixture solution. After further incubation for $30 \mathrm{~min}$ at $37^{\circ} \mathrm{C}$, the cells were washed three times, fixed, and stained with DAPI for imaging.

Measurement of intracellular ROS. 4T1 Cells were plated on a glass-bottomed plate. After cultured for $24 \mathrm{~h}$, LucCe6/Fuso-lip or Ce6 DPBS solution was added and incubated for 30 min or $2 \mathrm{~h}$, respectively. After washing twice with DPBS, a $50 \mathrm{nM}$ DCFDA/DPBS labeling solution was added into the cell culture and incubated for $30 \mathrm{~min}$ at $37^{\circ} \mathrm{C}$. For the BL-PDT group, CTZ $(100 \mu \mathrm{M})$ was added, and the cells were incubated for $4 \mathrm{~h}$. For the laser-PDT group, $405 \mathrm{~nm}$ laser was illuminated with a fluence of $1 \mathrm{~J} / \mathrm{cm}^{2}$. After completing the treatment, the cells were washed three times and fixed by $4 \%$ PFA for imaging. 
Assessment of therapeutic effects in vitro. 4T1 (5,000 cells per well) and MDA-MB-231 cells (10,000 cells per well) were seeded in 96-well plates and incubated for $24 \mathrm{~h}$. After washing with DPBS, the cells received Luc-Ce6/Fusolip at different concentrations and incubated for $1 \mathrm{~h}$ at $37^{\circ} \mathrm{C}$. The cells were washed with DPBS, received $100 \mu \mathrm{M}$ $\mathrm{CTZ}$, and incubated for $4 \mathrm{~h}$. The cells were then washed and then rested for $24 \mathrm{~h}$ at $37^{\circ} \mathrm{C}$. The viability of the cells was measured by using the CCK- 8 assay. Control cells underwent the same washing and incubation process without adding Luc-Ce6 and CTZ reagents. For laser-PDT, cells were incubated with a Ce6-HA DPBS solution for $2 \mathrm{~h}$ and then irradiated with a $405 \mathrm{~nm}$ laser for $1 \mathrm{~J} / \mathrm{cm}^{2}$. For imaging analysis of cell viability, a Live/Dead ${ }^{\mathrm{TM}}$ cell imaging kit (Invitrogen) was used. 4T1 cells were incubated for $24 \mathrm{~h}$, rinsed with DPBS, and stained with the live/dead kit for $15 \mathrm{~min}$ at room temperature. The cells were washed with DPBS and fixed by $4 \%$ PFA for imaging. For apoptosis assay, 4T1 cells were plated in 24-well plates at a density of $5 \times 10^{4}$ cells per well and cultured for $24 \mathrm{~h}$. After incubation with Luc-Ce6/Fuso-lip for $30 \mathrm{~min}$ or Ce6 for $2 \mathrm{~h}, 4 \mathrm{~T} 1$ cells were treated with $100 \mu \mathrm{M} \mathrm{CTZ}$ for BL-PDT and $405 \mathrm{~nm}$ laser $\left(1 \mathrm{~J} / \mathrm{cm}^{2}\right)$ for laser-PDT. After incubated for $24 \mathrm{~h}$, the cells were harvested, washed twice with cold DPBS, and then resuspended in DPBS buffer. The cell solution was then mixed and incubated with Annexin-V-FITC and PI (Abcam) working solution for $20 \mathrm{~min}$ at room temperature. Flow cytometry (BD FACSAria Cell Sorting System) was used to measure the apoptosis signals from the fluorescent probes.

Tissue penetration efficiency study in vitro. To measure the penetration depth of $405 \mathrm{~nm}$ laser, chicken breast tissue slices with a thickness of $50 \mu \mathrm{m}$ each was prepared through standard freezing tissues and cryosection. Each breast tissue slice was fully spread and put on a glass slide. The glass slides were stacked on top of each other to simulate thicker tissues ( $50 \mu \mathrm{m}$ for one slice to $300 \mu \mathrm{m}$ for 6 stacks). 4T1 cells were prepared in a plate and incubated with Ce6-HA for $2 \mathrm{~h}$. $405 \mathrm{~nm}$ laser light with a fluence $1 \mathrm{~J} / \mathrm{cm}^{2}$ was illuminated onto the first tissue slice on top. The optical attenuation through tissue stacks was measured by using an optical power meter (Thorlabs, PM400). After the laser-PDT, the cells were incubated for $24 \mathrm{~h}$, and their viability was measured by using the CCK8 assay.

In vivo tumor model. All animal studies and related protocols (2015N000205) have been reviewed and approved by the MGH Institutional Animal Care and Use Committee (IACUC) following the NIH guidelines. BALB/c female mice (5 6 weeks old) were purchased from Jackson Laboratories. A metastatic TNBC orthotopic model was produced by carefully injecting $\sim 10^{6} 4 \mathrm{~T} 1$ tumor cells into the mammary fat pad of a mouse. From the Week 1 following inoculation, the tumor volume was measured by external manual examination. The tumor volume was calculated as (length times width^2)/2. Each week post inoculation, tumor tissues, sentinel LNs (SLNs), and lungs were harvested to analyze the extent of tumor invasion and metastasis. Tissue sections were stained with Hematoxylin \& Eosin (H\&E) and antibodies against Ki-67 and epidermal growth factor receptor (EGFR).

In vivo biodistribution after intratumoral delivery. Mice with orthotopic 4T1 TNBC tumors were randomly divided into two groups ( $\mathrm{n}=3$ each). Fuso-lip stained with DiR dyes was used. A Luc-Ce6/Fuso-lip PBS solution was injected into the tumor at several locations to cover the entire volume of the tumor at $2.5 \mathrm{mg} / \mathrm{kg}$ dose (based on the Luc-Ce6 amount). Mice were sacrificed at $6 \mathrm{~h}$ after injection. The entire tumor was carefully collected and quickly frozen in OCT gel (Tissue-Tek ${ }^{\oplus}$ ) for cryosection. To obtain spatial distribution of Luc-Ce6 in the tumor, four tumor slices ( $8 \mu \mathrm{m}$ thick each) were cut with an inter-slice distance of $50 \mu \mathrm{m}$. The sham-treated control group received the same multipoint intratumoral injection of PBS. The tumor slices were imaged by using a fluorescence microscope (Keyence, BZ-X700).

In vivo antitumor efficacy. For initial assessment, 4T1 tumor bearing mice were randomly divided into 4 groups ( $n=3$ each), each receive PBS, Luc-Ce6/Fuso-lip only, BL-PDT, and laser-PDT. At Day 14 after tumor inoculation, 
Luc-Ce6/Fuso-lip PBS solution was directly injected into the tumor at multiple locations at $2.5 \mathrm{mg} / \mathrm{kg}$ dose. $50 \mu \mathrm{l}$ PBS was injected for the PBS group. For the BL-PDT group, $50 \mu \mathrm{CTZ}(100 \mu \mathrm{M})$ was intravenously injected into the tail vein at $6 \mathrm{~h}$ after the injection of Luc-Ce6/Fuso-lip, and additional CTZ with the same amount was administered every $4 \mathrm{~h}$ for total $24 \mathrm{~h}$ duration. For the laser-PDT group, the skin directly on top of the tumor was irradiated with $405 \mathrm{~nm}$ laser with a dose of $90 \mathrm{~J} / \mathrm{cm}^{2} .3$ days after treatment, the mice were euthanized, and the whole tumors were collected and fixed in 4\% PFA for 2 days. Then the tumor tissues were transferred for staining for H\&E, Ki-67, EGFR, and TUNEL following the standard protocol. The tissue slices were scanned by using a Nanozoomer Slide Scanner (Hamamatsu).

For testing early-stage tumor treatments, mice with orthotopic 4T1 tumors were randomly divided into 4 groups ( $n=5$ each). At Day 7 after tumor inoculation, Luc-Ce6/Fuso-lip PBS solution was injected into the tumor by 2.5 $\mathrm{mg} / \mathrm{kg}$ dose. Starting from $6 \mathrm{~h}$ after the injection, CTZ $(100 \mu \mathrm{M})$ was intravenously injected to the BL-PDT group 6 times with an interval of $4 \mathrm{~h}$. A single $405 \mathrm{~nm}$ laser irradiation $\left(90 \mathrm{~J} / \mathrm{cm}^{2}\right.$ at the skin) was given to the laser-PDT group. Following the treatments, the tumor size and body weight, as well as general health conditions, of the mice were measured every other day. 17 days after the completion of the treatment, the mice were euthanized. Sentinel lymph nodes (SLNs) and lungs were collected and fixed with 4\% PFA for 1 day and embedded into paraffin for sectioning. Tissue sections of the SLNs and lungs were stained with H\&E and antibodies against Ki-67 and EGFR. Additionally, various other organs, such as the heart, liver, kidney, and spleen, were also harvested, fixed by $4 \%$ PFA and examined by H\&E histology.

BL-PDT for neoadjuvant therapy. 2 weeks post tumor inoculation, mice with orthotopic 4T1 tumors were randomly divided into PBS control, Laser-PDT, and BL-PDT groups ( $\mathrm{n}=3$ each). For the laser-PDT and BL-PDT groups, Luc-Ce6/Fuso-lip-PBS solution $(1.0 \mathrm{mg} / \mathrm{kg}$ ) was carefully injected into the tumor boundaries in all sides using a find needle. For distribution measurement, $6 \mathrm{~h}$ after the injection, the tumor and its neighboring normal tissues were harvested and quickly frozen in OCT gel for cryo-sectioning. For testing neoadjuvant therapy, $50 \mu \mathrm{l}$ CTZ $(100 \mu \mathrm{M})$ was intravenously injected every $4 \mathrm{~h} 6$ times for the BL-PDT group. $90 \mathrm{~J} / \mathrm{cm}^{2}$ of $405 \mathrm{~nm}$ laser was given to the laser-PDT group. 5 days after treatment, tissues including the tumor boundary were harvested and fixed in 4\% PFA for staining for H\&E, Ki-67, EGFR, and TUNEL.

Statistical analysis. The data are presented as means \pm standard deviation, as noted in each case. The number of samples, $n$, are provided. Two-way analysis of variance (ANOVA) was used. $P<0.05$ was considered to indicate a statistically significant difference. $P<0.05, P<0.01$, and $P<0.001$ are indicated with single, double, and triple asterisks, respectively.

Data availability. The data that support the figures within this paper are available upon reasonable request.

\section{References}

1. Celli JP, Spring BQ, Rizvi I, Evans CL, Samkoe KS, Verma S, et al. Imaging and Photodynamic Therapy: Mechanisms, Monitoring, and Optimization. Chemical Reviews 2010, 110(5): 2795-2838.

2. Yun SH, Kwok SJJ. Light in diagnosis, therapy and surgery. Nature Biomedical Engineering 2017, 1(1): 0008.

3. Mitsunaga M, Ogawa M, Kosaka N, Rosenblum LT, Choyke PL, Kobayashi H. Cancer cell-selective in vivo near infrared photoimmunotherapy targeting specific membrane molecules. Nature Medicine 2011, 17(12): 1685-1692. 
4. Idris NM, Gnanasammandhan MK, Zhang J, Ho PC, Mahendran R, Zhang Y. In vivo photodynamic therapy using upconversion nanoparticles as remote-controlled nanotransducers. Nature Medicine 2012, 18(10): 1580-1586.

5. He JJ, Wang Y, Missinato MA, Onuoha E, Perkins LA, Watkins SC, et al. A genetically targetable near-infrared photosensitizer. Nature Methods 2016, 13(3): 263-271.

6. Huang HY, Yu BL, Zhang PY, Huang JJ, Chen Y, Gasser G, et al. Highly Charged Ruthenium(II) Polypyridyl Complexes as Lysosome-Localized Photosensitizers for Two-Photon Photodynamic Therapy. Angewandte Chemie-International Edition 2015, 54(47): 14049-14052.

7. Gao R, Mei X, Yan DP, Liang RZ, Wei M. Nano-photosensitizer based on layered double hydroxide and isophthalic acid for singlet oxygenation and photodynamic therapy. Nature Communications 2018, 9(1): 2798.

8. Kotagiri N, Sudlow GP, Akers WJ, Achilefu S. Breaking the depth dependency of phototherapy with Cerenkov radiation and low-radiance-responsive nanophotosensitizers. Nature Nanotechnology 2015, 10(4): 370-379.

9. $\mathrm{Xu} \mathrm{XQ}, \mathrm{An} \mathrm{HJ}$, Zhang DL, Tao H, Dou Y, Li XH, et al. A self-illuminating nanoparticle for inflammation imaging and cancer therapy. Science Advances 2019, 5(1): eaat2953.

10. Jeon J, You DG, Um W, Lee J, Kim CH, Shin S, et al. Chemiluminescence resonance energy transfer-based nanoparticles for quantum yield-enhanced cancer phototheranostics. Science Advances 2020, 6(21): eaaz8400.

11. Kim YR, Kim S, Choi JW, Choi SY, Lee SH, Kim H, et al. Bioluminescence-Activated Deep-Tissue Photodynamic Therapy of Cancer. Theranostics 2015, 5(8): 805-817.

12. Leechen SF, Yu CT, Wu DR, Jan KY. Differential effects of luminol, nickel, and arsenite on the rejoining of ultraviolet light and alkylation-induced DNA breaks. Environmental and Molecular Mutagenesis 1994, 23(2): 116-120.

13. Roda A, Guardigli M. Analytical chemiluminescence and bioluminescence: latest achievements and new horizons. Analytical and Bioanalytical Chemistry 2012, 402(1): 69-76.

14. Roda A, Guardigli M, Michelini E, Mirasoli M, Pasini P. Analytical bioluminescence and chemiluminescence. Analytical Chemistry 2003, 75(21): 462A-470A.

15. Kim S, Jo H, Jeon M, Choi MG, Hahn SK, Yun SH. Luciferase-Rose Bengal conjugates for singlet oxygen generation by bioluminescence resonance energy transfer. Chemical Communications 2017, 53(33): 45694572.

16. Kim EH, Park S, Kim YK, Moon M, Park J, Lee KJ, et al. Self-luminescent photodynamic therapy using breast cancer targeted proteins. Science Advances 2020, 6(37): eaba3009.

17. Loening AM, Fenn TD, Gambhir SS. Crystal structures of the luciferase and green fluorescent protein from Renilla reniformis. Journal of Molecular Biology 2007, 374(4): 1017-1028.

18. Loening AM, Fenn TD, Wu AM, Gambhir SS. Consensus guided mutagenesis of Renilla luciferase yields enhanced stability and light output. Protein Engineering Design \& Selection 2006, 19(9): 391-400.

19. Spikes JD, Bommer JC. Photosensitizing properties of mono-L-aspartyl chlorin e6 (NPe6): a candidate sensitizer for the photodynamic therapy of tumors. Journal of Photochemistry and Photobiology B-Biology 1993, 17(2): 135-143. 
20. Sadanala KC, Chaturvedi PK, Seo YM, Kim JM, Jo YS, Lee YK, et al. Sono-Photodynamic Combination Therapy: A Review on Sensitizers. Anticancer Research 2014, 34(9): 4657-4664.

21. Kay A, Humphrybaker R, Gratzel M. Artificial Photosynthesis. 2. Investigations on the Mechanism of Photosensitization of Nanocrystalline $\mathrm{TiO}_{2}$ Solar Cells by Chlorophyll Derivatives. Journal of Physical Chemistry 1994, 98(3): 952-959.

22. Ragas X, Jimenez-Banzo A, Sanchez-Garcia D, Batllori X, Nonell S. Singlet oxygen photosensitisation by the fluorescent probe Singlet Oxygen Sensor Green (R). Chemical Communications 2009(20): 2920-2922.

23. Kim B, Pang HB, Kang J, Park JH, Ruoslahti E, Sailor MJ. Immunogene therapy with fusogenic nanoparticles modulates macrophage response to Staphylococcus aureus. Nature Communications 2018, 9(1): 1969.

24. Zuris JA, Thompson DB, Shu Y, Guilinger JP, Bessen JL, Hu JH, et al. Cationic lipid-mediated delivery of proteins enables efficient protein-based genome editing in vitro and in vivo. Nature Biotechnology 2015 , 33(1): 73-80.

25. Guan J, Shen Q, Zhang Z, Jiang ZX, Yang Y, Lou MQ, et al. Enhanced immunocompatibility of ligandtargeted liposomes by attenuating natural IgM absorption. Nature Communications 2018, 9(1): 2982.

26. Zhao F, Zhao Y, Liu Y, Chang XL, Chen CY, Zhao YL. Cellular Uptake, Intracellular Trafficking, and Cytotoxicity of Nanomaterials. Small 2011, 7(10): 1322-1337.

27. Behzadi S, Serpooshan V, Tao W, Hamaly MA, Alkawareek MY, Dreaden EC, et al. Cellular uptake of nanoparticles: journey inside the cell. Chemical Society Reviews 2017, 46(14): 4218-4244.

28. Wu CY, Wu YF, Jin Y, Zhu PY, Shi WW, Li JL, et al. Endosomal/lysosomal location of organically modified silica nanoparticles following caveolae-mediated endocytosis. Rsc Advances 2019, 9(24): 13855-13862.

29. Choi SI, Maeng YS, Kim TI, Lee Y, Kim YS, Kim EK. Lysosomal Trafficking of TGFBlp via Caveolae-Mediated Endocytosis. Plos One 2015, 10(4): e0119561.

30. Biswas R, Moon JH, Ahn JC. Chlorin e6 Derivative Radachlorin Mainly Accumulates in Mitochondria, Lysosome and Endoplasmic Reticulum and Shows High Affinity toward Tumors in Nude Mice in Photodynamic Therapy. Photochemistry and Photobiology 2014, 90(5): 1108-1118.

31. Hamblin MR, Miller JL, Rizvi I, Ortel B, Maytin EV, Hasan T. Pegylation of a chlorin(e6) polymer conjugate increases tumor targeting of photosensitizer. Cancer Research 2001, 61(19): 7155-7162.

32. Kessel D, Oleinick NL. Cell Death Pathways Associated with Photodynamic Therapy: An Update. Photochemistry and Photobiology 2018, 94(2): 213-218.

33. Cinkaya A, Akin M, Sengul A. Evaluation of treatment outcomes of triple-negative breast cancer. Journal of Cancer Research and Therapeutics 2016, 12(1): 150-154.

34. Bourgeois-Daigneault MC, Roy DG, Aitken AS, El Sayes N, Martin NT, Varette O, et al. Neoadjuvant oncolytic virotherapy before surgery sensitizes triple- negative breast cancer to immune checkpoint therapy. Science Translational Medicine 2018, 10(422): eaao1641.

35. Cortazar P, Geyer CE. Pathological Complete Response in Neoadjuvant Treatment of Breast Cancer. Annals of Surgical Oncology 2015, 22(5): 1441-1446.

36. Li XS, Kolemen S, Yoon J, Akkaya EU. Activatable Photosensitizers: Agents for Selective Photodynamic Therapy. Advanced Functional Materials 2017, 27(5) : 1604053. 
37. He CB, Duan XP, Guo NN, Chan C, Poon C, Weichselbaum RR, et al. Core-shell nanoscale coordination polymers combine chemotherapy and photodynamic therapy to potentiate checkpoint blockade cancer immunotherapy. Nature Communications 2016, 7: 12499.

38. Yan H, Shang WT, Sun XD, Zhao LY, Wang XM, Zhang SX, et al. Neoadjuvant nano-photothermal therapy used before operation effectively assists in surgery for breast cancer. Nanoscale 2019, 11(2): 706-716.

39. Liu CH, Cao Y, Cheng YR, Wang DD, Xu TL, Su L, et al. An open source and reduce expenditure ROS generation strategy for chemodynamic/photodynamic synergistic therapy. Nature Communications 2020, 11(1): 1735.

40. Yu GC, Yu S, Saha ML, Zhou J, Cook TR, Yung BC, et al. A discrete organoplatinum(II) metallacage as a multimodality theranostic platform for cancer photochemotherapy. Nature Communications 2018, 9(1): 4335.

41. Yu ZZ, Zhou P, Pan W, Li N, Tang B. A biomimetic nanoreactor for synergistic chemiexcited photodynamic therapy and starvation therapy against tumor metastasis. Nature Communications 2018, 9(1): 5044.

42. Zhao ZM, Ukidve A, Kim J, Mitragotri S. Targeting Strategies for Tissue-Specific Drug Delivery. Cell 2020, 181(1): 151-167.

43. van der Meel R, Sulheim E, Shi Y, Kiessling F, Mulder WJM, Lammers T. Smart cancer nanomedicine. Nature Nanotechnology 2019, 14(11): 1007-1017.

44. Anselmo AC, Gokarn Y, Mitragotri S. Non-invasive delivery strategies for biologics. Nature Reviews Drug Discovery 2019, 18(1): 19-40.

45. Gurbatri CR, Lia I, Vincent R, Coker C, Castro S, Treuting PM, et al. Engineered probiotics for local tumor delivery of checkpoint blockade nanobodies. Science Translational Medicine 2020, 12(530): eaax0876.

46. Wang YC, Wang Y, Wang YG, Murray CK, Hamblin MR, Hooper DC, et al. Antimicrobial blue light inactivation of pathogenic microbes: State of the art. Drug Resistance Updates 2017, 33: 1-22.

47. Hui J, Dong PT, Liang LJ, Mandal T, Li JJ, Ulloa ER, et al. Photo-Disassembly of Membrane Microdomains Revives Conventional Antibiotics against MRSA. Advanced Science 2020, 7(6): 1903117.

\section{Acknowledgements}

This work was supported by US National Institutes of Health $(\mathrm{NIH})$ grant, 5R01CA192878. We thank Drs. Tayyaba Hasan and Seonghoon Kim for contributions in the initial stage of the project, and Dr. Jenny Zhao and the Wellman Photopathology Core for helping with tissue sectioning, staining, and imaging.

\section{Author contributions}

H.Y. and S.H.Y. designed the experiments. H.Y. and K.H.K. fabricated Luc-Ce6 conjugates. H.Y. and S.F. developed fusion liposomal delivery. H.Y. and A.K. performed biocompatibility assays. Y.W. and J.H. assisted in chemistry. H.Y. conducted in vitro experiments. H.Y. and S.F. established the mouse model. H.Y. performed in vivo experiments. H.Y. and S.H.Y. prepared figures and wrote the manuscript, with inputs from other co-authors.

\section{Competing interests}

Authors have no competing interests. 
$\mathbf{a}$

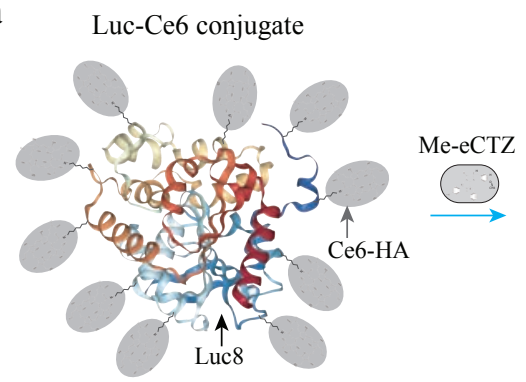

b

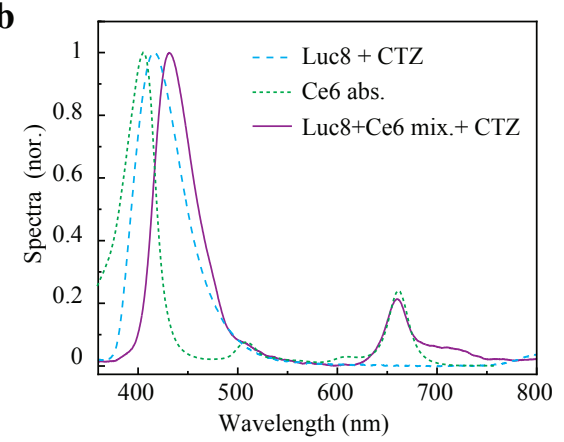

e

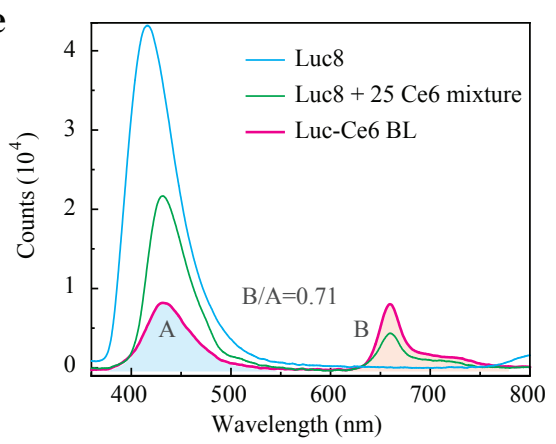

BL energy by Luc-CTZ reaction

c

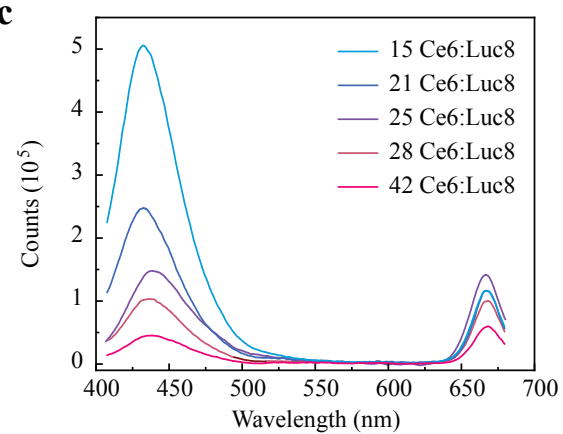

$\mathbf{f}$

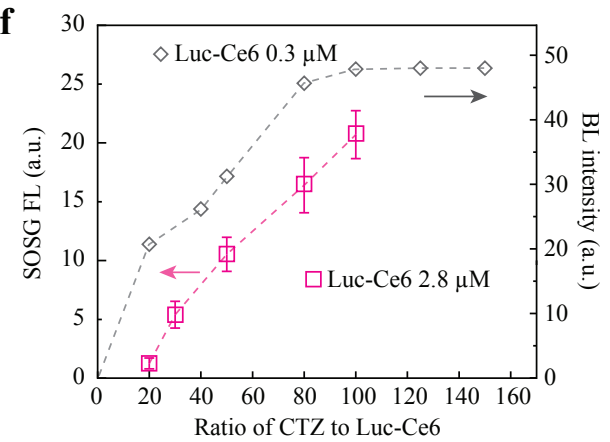

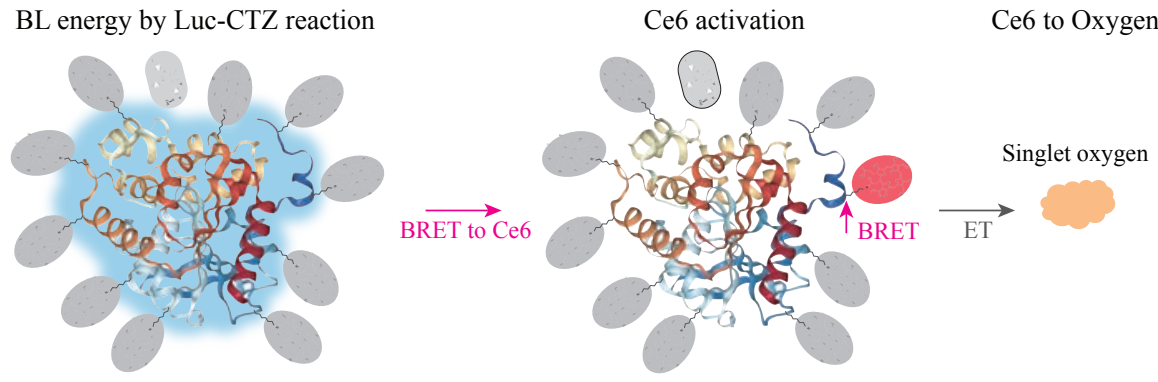

Ce6 activation

Ce6 to Oxygen

d

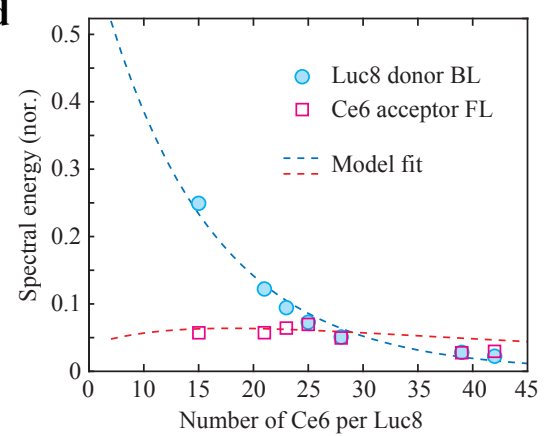

g

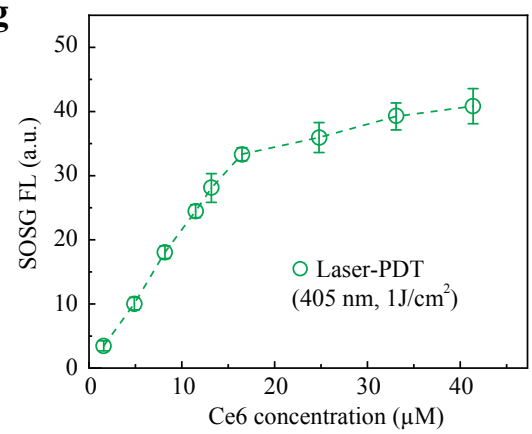

Figure 1. Design and physicochemical characterization of the Luc-Ce6 conjugate. (a) Schematic of the structure of Luc-Ce6 conjugate and its working principle. A Ce6 molecule receives resonant energy transfer from the complex of a RLuc8 protein (Luc) and substrate methoxy e-coelenterazine (Me-eCTZ). (b) Normalized BL spectrum of Luc (blue dashed line), absorption spectra of Ce6-HA (green dashed line), and BL spectra of the mixture of Luc and Ce6-HA (the ratio is 1:25) (purple). (c) BL spectra of Luc-Ce6 conjugates with different mole conjugation ratios of Ce6-HA molecules to Luc protein. (d) The dependence of spectral energy for the donor and acceptor on the number of Ce6 molecules per Luc8. (e) BL spectra of Luc8 protein ( $0.3 \mu \mathrm{M})$, the mixture of Luc (0.3 $\mu \mathrm{M})$ and Ce6-HA (7.5 $\mu \mathrm{M})$, and Luc-Ce6 conjugate (0.3 $\mu \mathrm{M}$ for Luc). A: donor emission (400-600 nm) area, B: acceptor emission (600-800 nm) area. (f) Changes in the BL intensity and SOSG fluorescence intensity as the proportion of substrate CTZ is varied at two different concentrations of Luc-Ce6. (g) SOSG fluorescence intensity as a function of Ce6 concentration in solution after laser-PDT (405 nm, $\left.125 \mathrm{~mW} / \mathrm{cm}^{2}, 8 \mathrm{~s}\right)$. 
$\mathbf{a}$

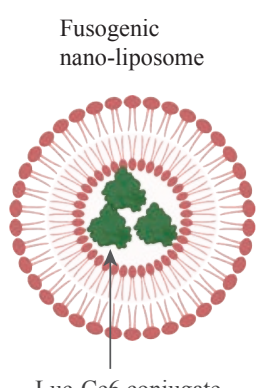

Luc-Ce6 conjugate

c

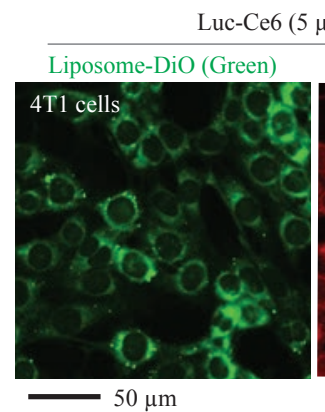

$\mathbf{e}$
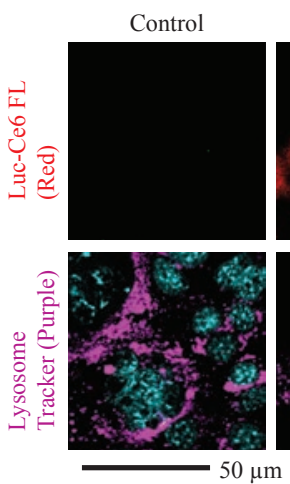

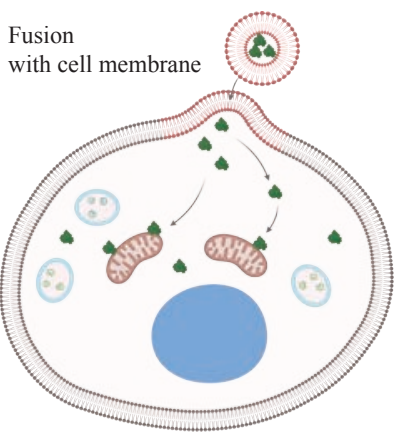

b

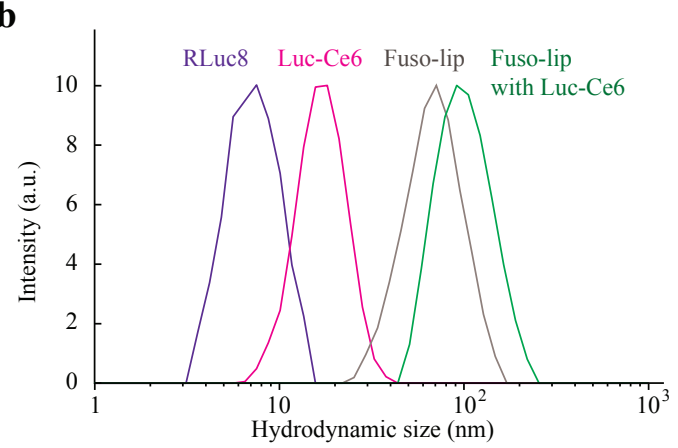

d 100

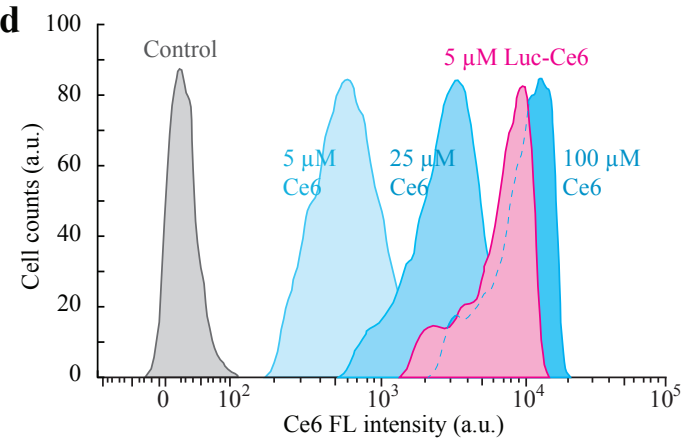

f

$60 \mathrm{~min}$
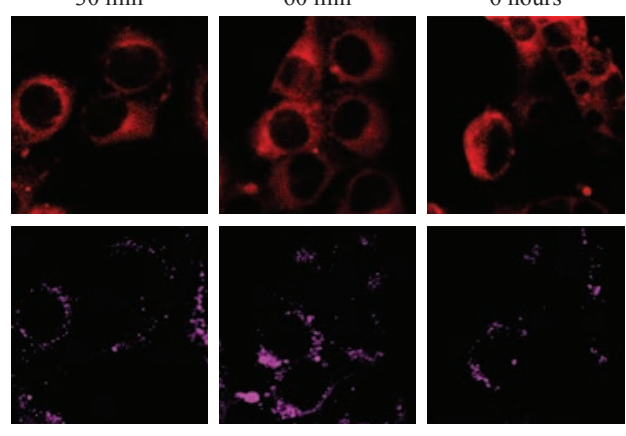

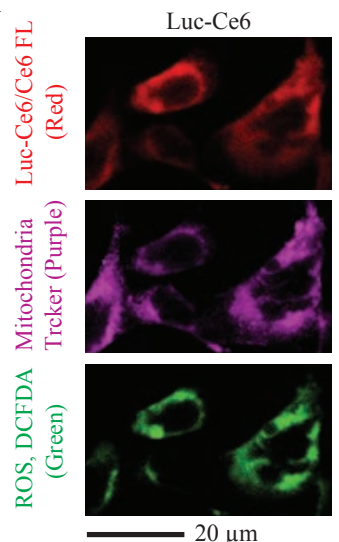

Ce6

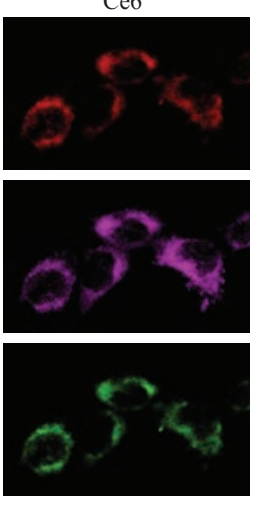

Figure 2. Intracellular delivery of Luc-Ce6 conjugates. (a) Schematic illustration of intracellular delivery. LucCe6 loaded in fusogenic nanoliposomes (Fuso-lip) enters the cell after Fuso-lip is fused with the cell membrane. Luc-Ce6 is in the cytoplasm produces ROS after reacting with CTZ. (b) The hydrodynamic sizes of the Luc protein, Luc-Ce6 conjugate, Fuso-lip, and Luc-Ce6/Fuso-lip. The Luc-Ce6 is about $17 \mathrm{~nm}$ in DPBS buffer, and the final Fusolip with Luc-Ce6 is about $100 \mathrm{~nm}$. (c) Fluorescence images of 4T1 cells after incubation with $5 \mu \mathrm{M}$ Luc-Ce6 (red)/Fuso-lip (green) for 30 min, or Ce6-HA molecules ( $25 \mu \mathrm{M}$ ) for $2 \mathrm{~h}$. (d) Flow cytometry data of cells incubated with $5 \mu \mathrm{M}$ Luc-Ce6/Fuso-lip and Ce6-HA with different concentrations. (e) Confocal fluorescence images of 4T1 cells after incubation with Luc-Ce6 (red)/Fuso-lip over time. The lysosomes were stained by Lysosome Tracker (purple). (f) Confocal fluorescence images of Luc-Ce6/Fuso-lip-treated and Ce6-treated 4T1 cells. Mitochondria (purple) were stained using a Mitochondria tracker kit, and intracellular ROS (green) was stained using $2^{\prime}, 7^{\prime}-$ dichlorofluorescein diacetate (DCFDA). 


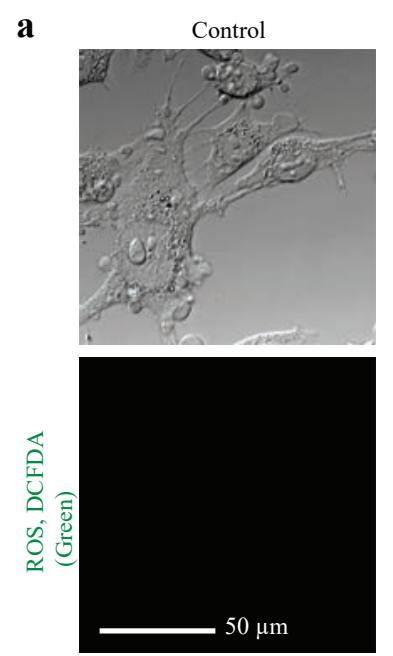

C
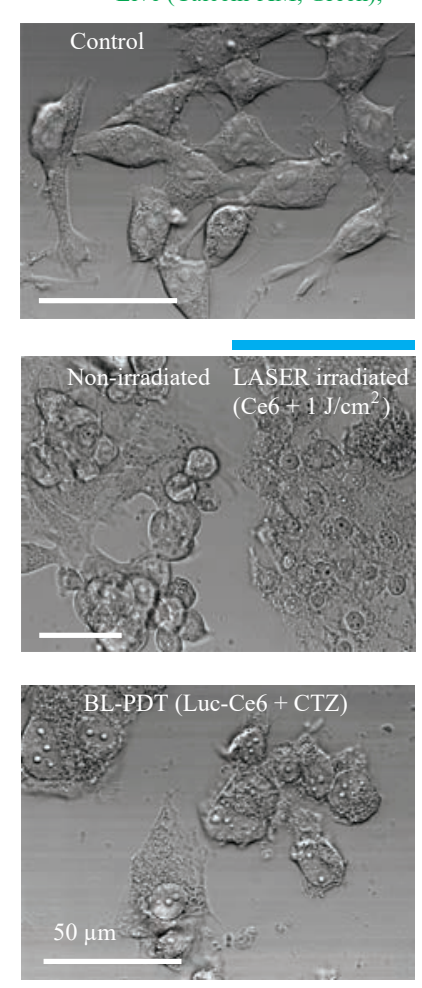

Luc-Ce6 $(10 \mu \mathrm{M})+\mathrm{CTZ}$
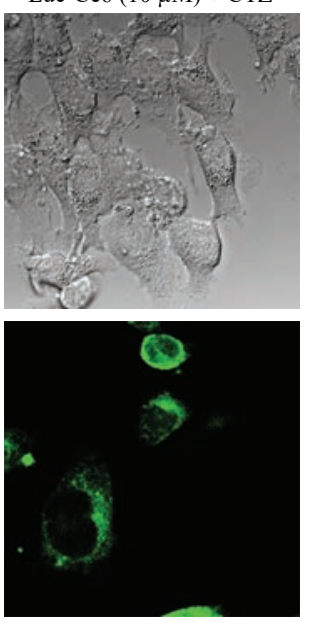

Dead (BOBO-3 Iodide, Red)
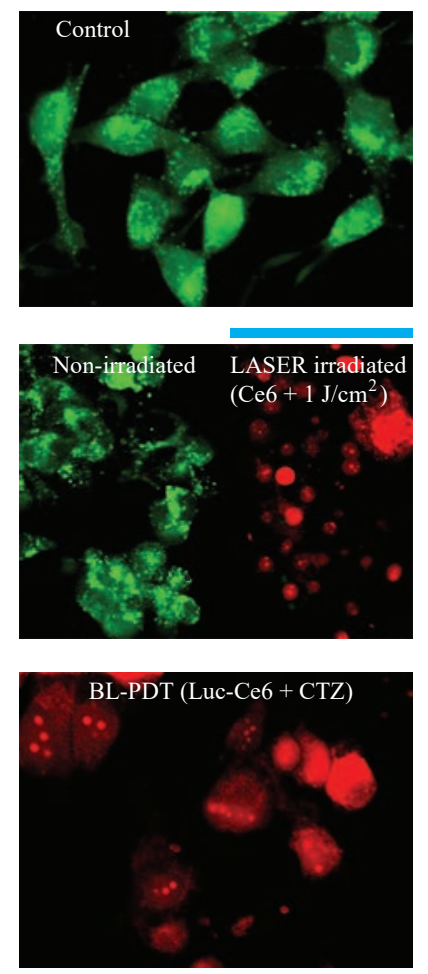

b
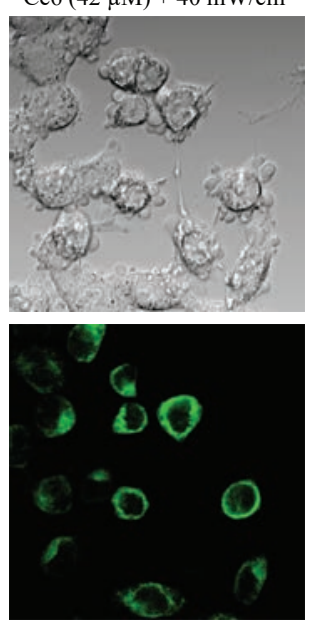

d

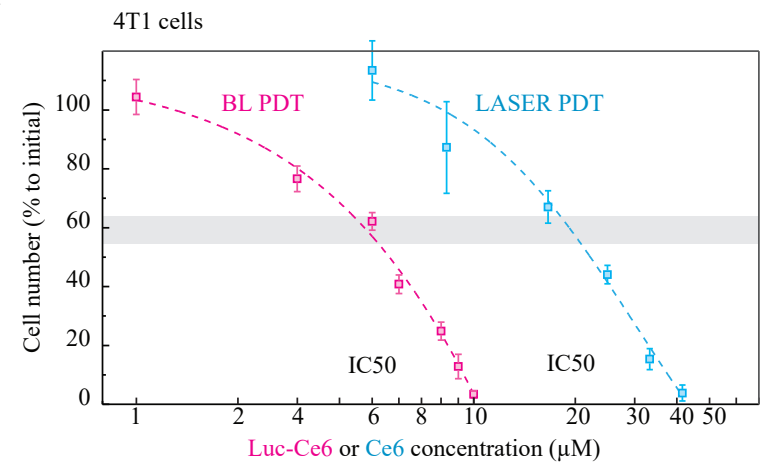

e

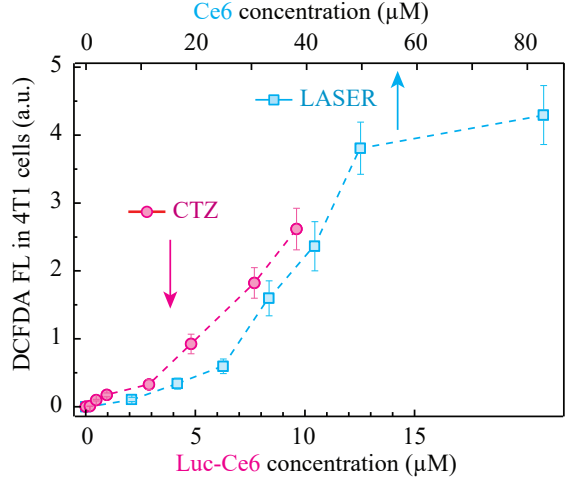

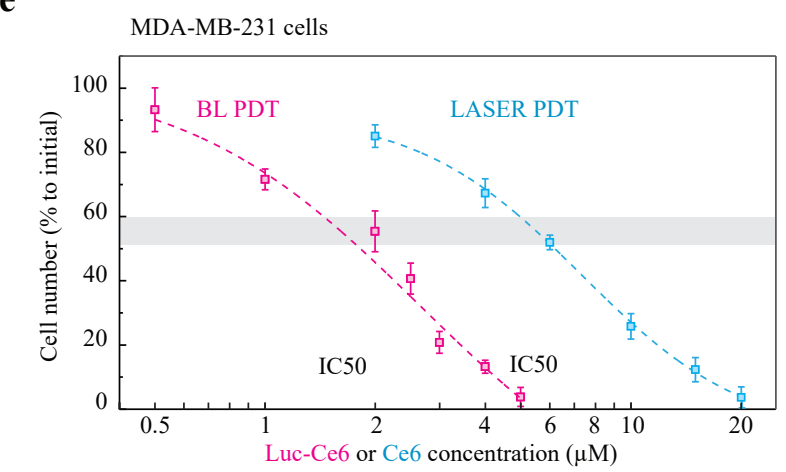

Figure 3. In vitro assessment of Luc-Ce6 based BL-PDT. (a) Confocal microscopy images of intracellular ROSsensing CDFDA (green) in 4T1 cells after BL-PDT (Luc-Ce6 $10 \mu \mathrm{M}$ and CTZ $100 \mu \mathrm{M}$ ) and laser-PDT (Ce6 and $405 \mathrm{~nm}$ laser, $1 \mathrm{~J} / \mathrm{cm}^{2}$ ). (b) Measured DCFDA intensity for BL-PDT and laser-PDT $\left(1 \mathrm{~J} / \mathrm{cm}^{2}\right)$ for various concentrations of LucCe6 and Ce6. (c) Fluorescence images of 4T1 cells stained with Calcein AM (live cells, green) and BOBO-3 lodide (dead cells, red). Scale bars, $50 \mu \mathrm{m}$. (d-e) Relative viability of 4T1 (d) and MDA-MB-231 cells (e) after BL-PDT and laser-PDT for different concentrations of Luc-Ce6 and Ce6. The horizontal grey band indicate the half-maximal inhibitory concentration $\left(\mathrm{IC}_{50}\right)$ levels. 
a

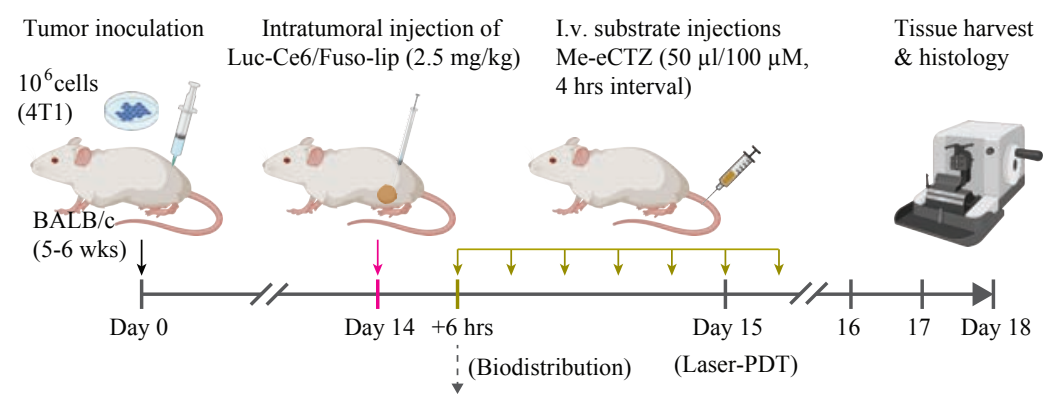

b

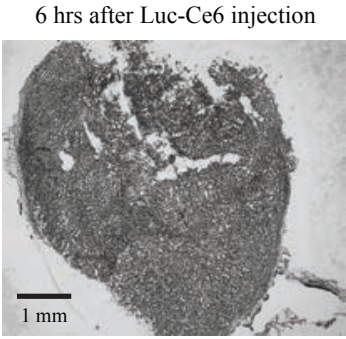

d
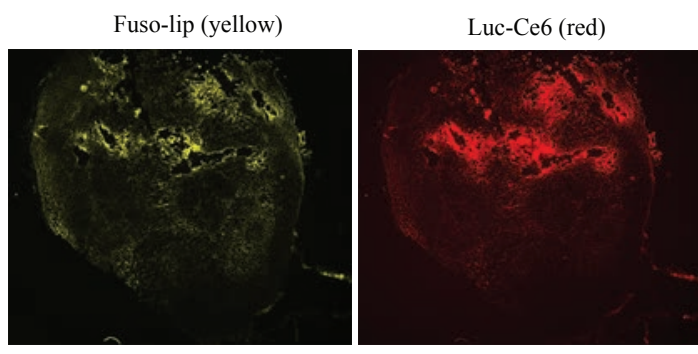

e

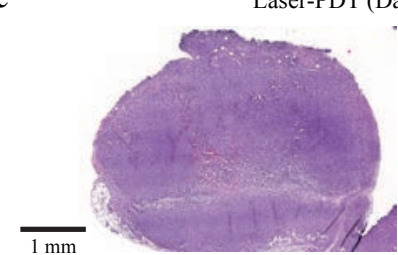

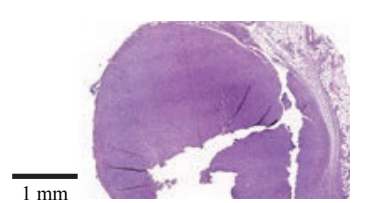

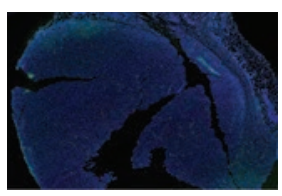

c
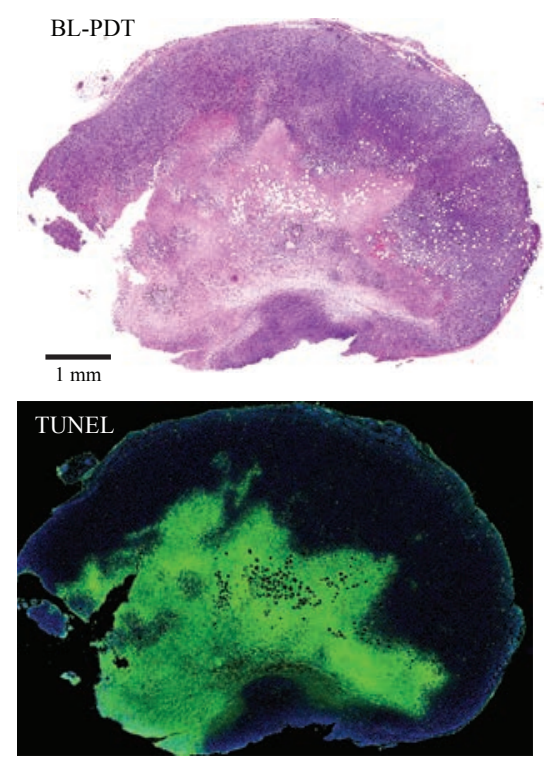

Laser-PDT (Day 15, $90 \mathrm{~J} / \mathrm{cm} 2)$

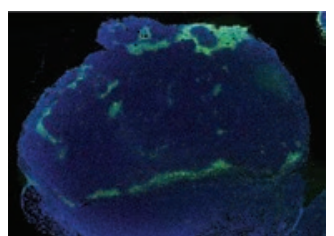

Figure 4. In vivo assessment of BL-PDT in a 4T1 TNBC orthotopic model. (a) Schematic representation of the treatment schedule for 4T1 tumors at the advanced stage. (b) Fluorescence microscopy images of the whole tumor cryosection from the Luc-Ce6/Fuso-lip-treated mice. Fuso-lip: yellow, Luc-Ce6: red, BF: bright field. (c) H\&E and immunofluorescence images of tumor sections after BL-PDT treatment. Green: TUNEL, blue: DAPI for nuclei. (d) H\&E and TUNEL-stained tumor sections after PBS injection. (e) H\&E and TUNEL-stained tumor sections after laser-PDT (405 nm, $\left.90 \mathrm{~J} / \mathrm{cm}^{2}\right)$. 


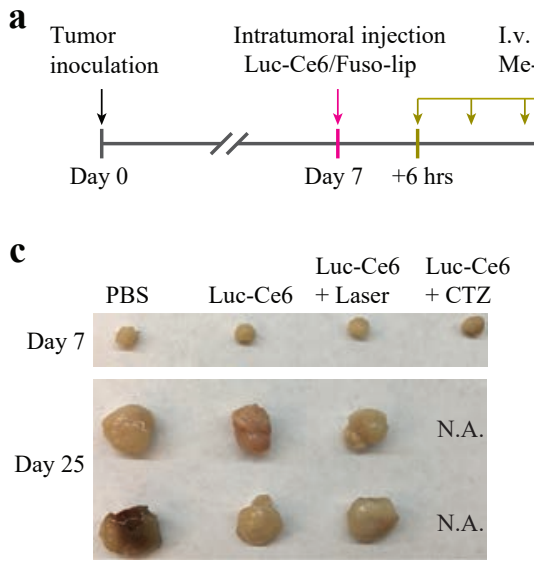

e

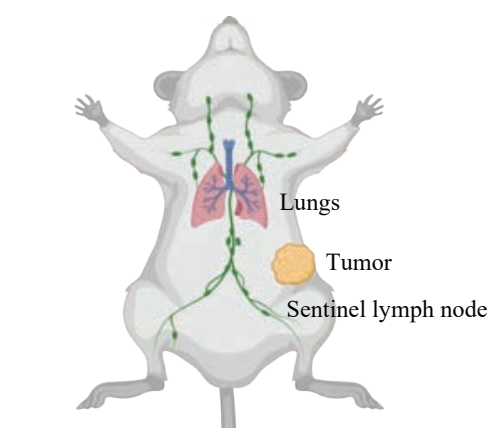

g
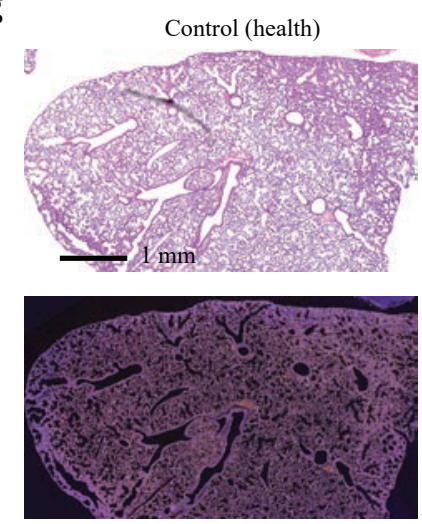

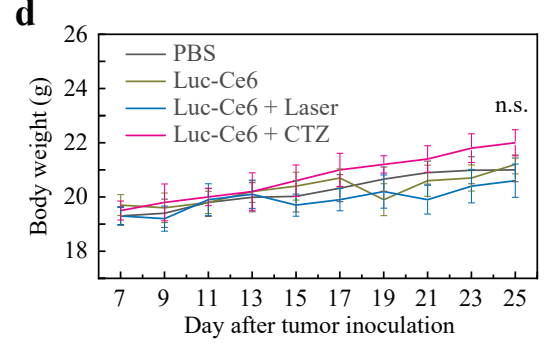

f

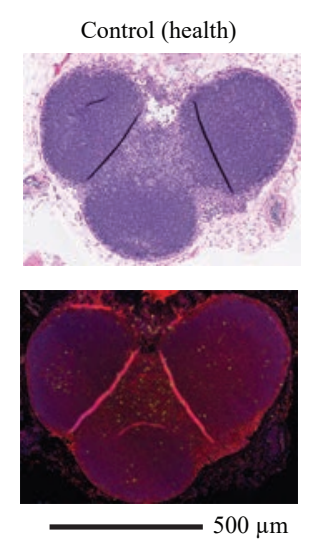

Sham (PBS)
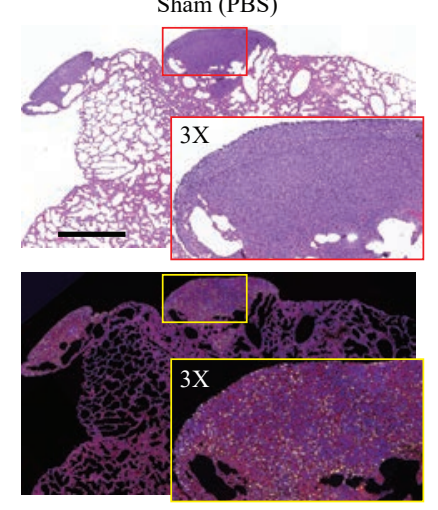

b
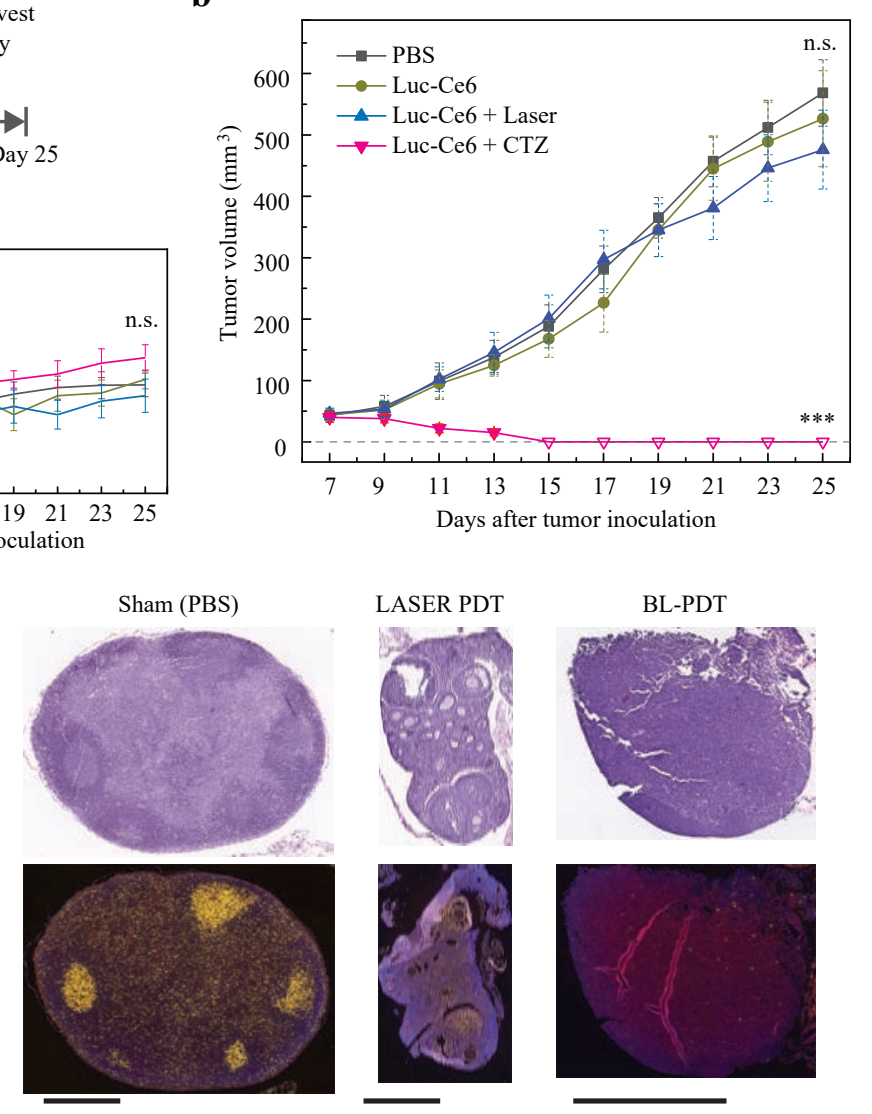

LASER PDT
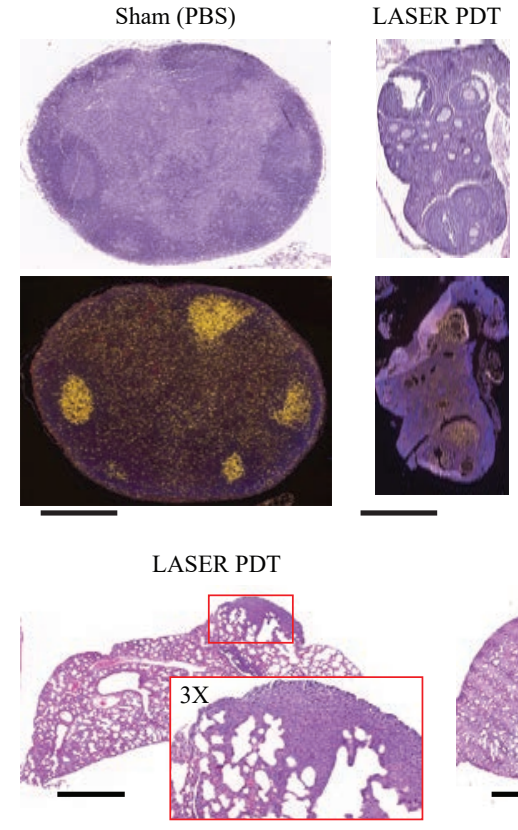

BL-PDT
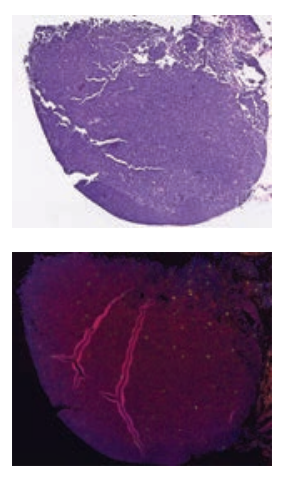

BL-PDT

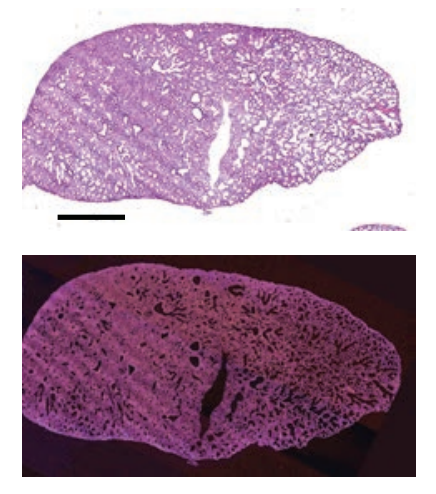

Figure 5. The effects of BL-PDT on 4T1 tumor growth. (a) Treatment schedule. (b) Growth curves of tumors in different groups: PBS injection only, Luc-Ce6 injection only, Luc-Ce6 and laser-PDT (405 nm, $\left.90 \mathrm{~J} / \mathrm{cm}^{2}\right)$, and BLPDT. Error bars are the standard deviation of 5 animals per group. ${ }^{* * *}, \mathrm{P}<0.001$; n.s.: not significant. (c) Photos of tumors harvested from different groups at different times. (d) Body weight. (e) Schematic showing the orthotopic tumor and sentinel lymph node. (f-g) H\&E histology and immunofluorescence (IF)-stained sentinel lymph nodes (f) and lungs (g) from breast tumor-bearing mice 18 days after treatment: unperturbed control, PBS injection only (sham), laser-PDT and BL-PDT. 4T1 cancer cells were stained by Ki-67 (cancer cell nucleus, yellow), EGFR (cell membrane, red), and DAPI (nucleus, blue). While metastasis is apparent in the lymph nodes and lungs in the PBS and laser-PDT groups, no evident metastasis was found in the control and BL-PDT groups. 
$\mathbf{a}$

BL-PDT along the tumor boundary

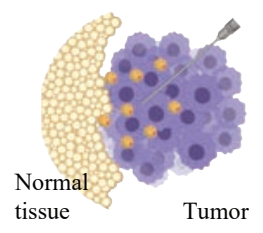

Tumor shinkage and margin demarcation

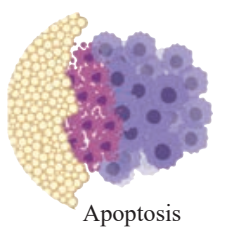

TUNEL (green)

c

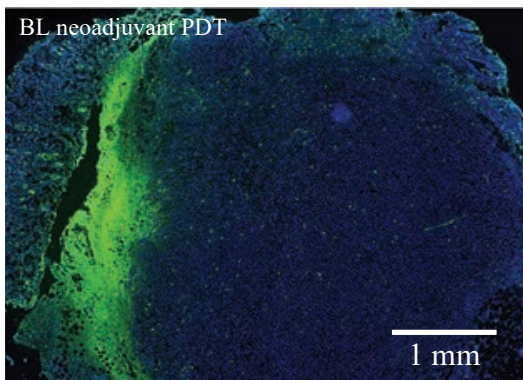

d

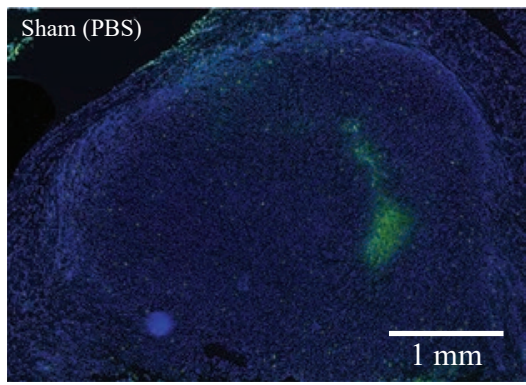

b 4T1 tumor bearing BALB/c mouse

Injection of Luc-Ce6/Fuso-lip along the boundary $(1.0 \mathrm{mg} / \mathrm{kg})$
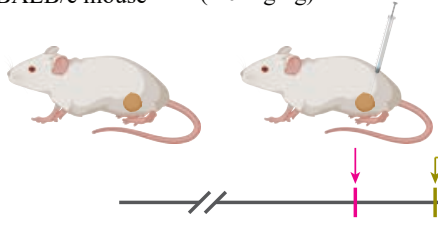

I.v. substrate injections Me-eCTZ $(50 \mu 1 / 100 \mu \mathrm{M}$, $4 \mathrm{hrs}$ interval)

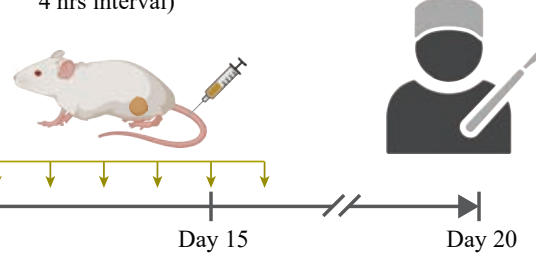

Ki-67 (yellow); EGFR (purple)

$H \& E$
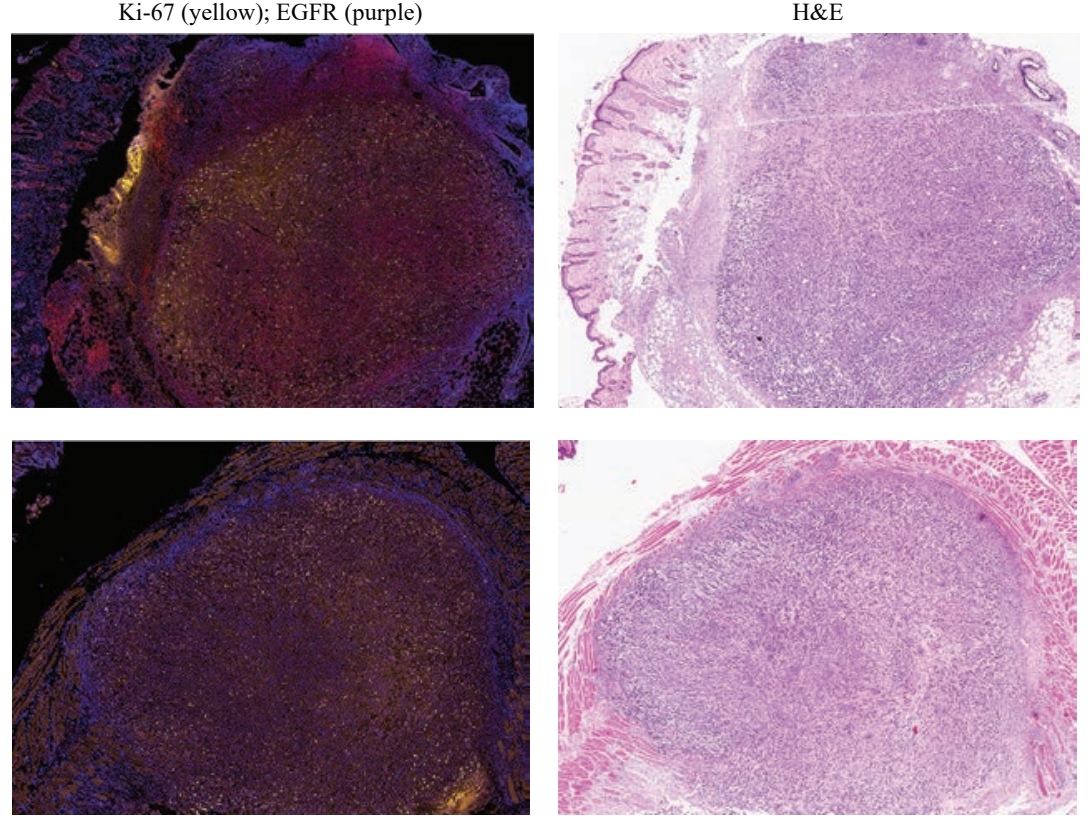

Figure 6. In vivo evaluation of BL-PDT for neoadjuvant therapy. (a) Neoadjuvant BL-PDT of an advanced primary tumor. (b) The treatment schedule of neoadjuvant BL-PDT for $4 \mathrm{~T} 1$ orthotopic tumor model. (c-d) TUNEL, H\&E, and immunofluorescence (IF)-stained tumor sections, including surrounding normal tissues, five days after (c) BL-PDT and (d) sham (PBS) treatment. TUNEL: green; Ki-67: yellow; EGFR: purple. 